\title{
Ontology of the Development Strategies: (The) Basis for Decision Support in Government Development Funds
}

\author{
Saša ARSOVSKI, Branko MARKOSKI, Nikola PETROV, Sanja STANISAVLJEV, Mila ZAKIN
}

\begin{abstract}
This paper presents a methodical approach to the creation of the ontological model which serves as a knowledge base in the decision-making process of evaluation clients who are applying for loans and subsides of state development funds. The role of the proposed ontological model is steering government subsidies and investments in industries and areas of socio - economic development which are defined by the state development strategies. The ultimate aim of this research is to suggest appropriate classifiers (priority indexes) for each ensemble of the socio-economic parameters in the context of scoring government development funds clients. The novelty of the research presented in this paper is reflected by the identifying and semantically presenting of social and economic characteristics, which covers the area of operation of state development funds and which are relevant for the development of the analyzed region. The advantages which are achieved by using the proposed ontology model are: decision-making process that excludes the subjective reasoning of the administrative staff and decision proposals which are justified in state development strategies. The authors in this paper propose an implementation procedure and show a prototype example of utilization of the created ontology.
\end{abstract}

Keywords: decision support systems; government development funds; ontologies; strategic planning

\section{INTRODUCTION}

Standard bank methodologies concerning client categorization and scoring include: probit analysis, nonparametric methods, neural networks or conditionindependent models [1], [15]. The credit-strategy focuses almost completely on predicting client behavior (score cards) based on their individual characteristics. In situations of data shortage on the client (or situations of special regulatory demands - borderline cases), the experts of the field are needed to assess the problem at hand and make a subjective decision.

State development funds are institutions established by various authorities (state, province, or local autonomy), which focus on supporting the development of small and medium enterprises whilst minimizing the costs of risks and transactions (e.g. of giving loans) [16], [18].

The individual characteristics of clients which are assessed using standard banking methods are just part of the decision-making criterion, and should be paired with broader social demands which should be met by state funds (regional, social and developmental). Because of this, the standard banking methods for client assessment are insufficient for decision making in state development funds.

The motivation for creating the ontological model presented in this paper is creating a general principle for scoring clients, the basis of which is steering state subventions into industrial and socio-economic development defined by state development strategies. This ontology has been named SCORE and will be further described.

By using the SCORE ontology in the decisionmaking process, decision proposal would be based on development strategies which have been implemented into the SCORE ontology.

The proposed ontology should not be seen as a basis for decision-making support of development politics for a specific region, but rather as a knowledge base of already accepted state development politics and results obtained from socio-economic analyses of the relevant domain.
The paper is organized as follows: the second section provides an overview of the related work. The third section presents conceptual model of the SCORE ontology. The fourth section presents stages of the ontology implementation. The fifth section shows the prototype example of the ontology use. In the final, sixth section, concluding remarks and directions for further research are given.

\section{RELATED WORK}

Decision support systems (DSS) are information systems which support users in the decision-making process. The authors in [2] generally identify three phases of the development process [2]:

1) Defining the decision problem

2) Gathering, storage and fusion of problem-specific data

3) Justification of the data needed in order to make a decision.

The ontologically governed DSS represents a systematic method of defining the process of decision making by utilizing ontology as its basis [3]. A standardized implementation procedure for decisionmaking does not yet exist.

The authors in [4] propose the use of ontology in the DSS domain of human resource management. The authors propose an integration of multiple data resources in order to support decision-making and improve predictions and projections of human potential supply and demand.

In [19] authors propose approach towards modeling a classical expert system using an ontology-based solution. Authors present a hierarchical rule-based system for the evaluation of reference ECG signals called the Minnesota Code [20]. The Minnesota Code is a classification system for the electrocardiogram that utilizes a defined set of measurement rules to assign specific numerical codes according to severity of the ECG (Electrocardiography) findings.

An ontology model-based representation presented in this paper provides the possibility to clearly define the 
relationships between diagnostic rules and inputs using axioms. Extensions to the diagnostic model are possible by introducing new concepts or axioms between concepts. Based on the original definitions of the Minnesota Code, the elements used for constructing the ontology rules are the waveforms, ECG leads, waveform value states and the grouping concepts. Authors present the ontology model of the Minnesota Code which is divided into 4 main groups: Sample, ECG leads, Waveforms and Rules.

In SCORE ontology, which is proposed in this paper, we have not been able to use rules because the input data are not strictly defined numerical values so that we proposed decision support system that relies on the ontological reasoning.

The authors in [17] present a methodology for building an ontologically controlled DSS for system government revision support.

Authors in [5] elaborate on the benefits of using ontologies for data representation as a building block for DSS systems. The authors also propose ontology architecture (knowledge basis) for the support of the decision-making procedure. The work describes three main components of the ontology: Problem, Data and Conclusion. The relations defined between basic ontology components are: hasData, hasConclusion and ProduceConclusion

The Problem component formally describes all aspects of the problem which the user can inscribe into the knowledge base. Generally, his component describes different attributes of the same problem. The Data component formally describes data which can be accessed and manipulated by the decision-making support system.

The Conclusion component describes conclusions such as: warnings, recommendations and suggestions. These conclusions are met on the basis of data in the Data component of the ontology.

Research that encompasses the creation of methods for defining estimation indicators for development of economical politics is described in [6] where the authors present results of research conducted in the CAVALA project. In this project, the implementation of decisionmaking tools is proposed as a crucial prerequisite for ontology creation. The paper describes phases preceding the creation of the ontology.

The authors identify three levels of knowledge used when modeling the ontology:

1) Regulation level - describes the need for clearly defining norms, procedures and prerequisites with the help of which it is possible to define criteria needed to assess the suitability of an investment and define a list of priorities (territory, projects or MSP) which will be supported. This level of knowledge is the most concrete level and generally influences the final level of the ontology,

2) Principle level - this level of knowledge represents conceptual norm level. This level represents typologies, categories and reasons for acting which are related to basic units (e.g. companies). This level of analyzed knowledge is used to create the middle level of the ontology.

3) Doxailevel - this level represents the paradigms of choice and visions in defining the norms or principles. In the context of the CAVALA project, this level of knowledge represents political decisions.

Because the SCORE ontology does not nurture ambitions of being a decision-making and developmentpolitics assessment support basis for a specific region, but rather a knowledge database of already accepted government development politics and results obtained by socio-economic analysis of the relevant domain, only the aspects of regulatory (normative) knowledge given in [6] are implemented in the SCORE ontology.

\section{SCORE ONTOLOGY}

The Methontology methodology was used for building ontology [9]. Methontology is one of the oldest methodologies for creating ontologies, sufficiently detailed, covers the entire life cycle and contains a phase of ontology integration. The Protégé editor [10] was used for ontology formalization. For the representation of the SCORE ontology we use OWL [11] and the SPARQL [12] is used as query language. The Pellet [13] is used for reasoning.

\subsection{Ontology Specification}

The SCORE ontology encompasses conceptualized knowledge of socio-economic characteristics of the region Autonomous province of Vojvodina (APV) and development strategies to which the development funds are supposed to contribute. In this way, the ontology supports the information system during the decision proposal creating process.

Identification of development characteristics of the analyzed domain. The domain analysis includes socioeconomic characteristics of the APV region and state development strategies for the given region. Two main documents ([7] and [8]) were used for the analysis. Research in the CODEX project refers to rural areas in the APV region and, amongst other things, includes the analysis of strategic development parameters of the mentioned regions.

Document [8] represents a good basis for modeling SCORE ontology concepts, because the activities held by the government development funds in APV are mainly directed towards supporting rural area development.

During the analysis and consideration of the socioeconomic aspects of undeveloped and marginalized areas in APV [8], the national legislation, as well as directives and strategic preferences of the relevant development strategies were taken into consideration.

The analysis of literature [7] identifies three main groups of socio-economic characteristics of the APV region:

a) Demographic characteristics include the population, education structure, age structure, activity and employment of citizens, as well as the gender structure in economic activities.

b) Economical characteristics encompass economic structure, agriculture, tourism, forestry, the refinement industry and the structure of agricultural husbandry.

c) Social characteristics include infrastructure and social service analysis. Based on the results of [8], the 
infrastructure can be divided into three main groups: traffic, water economy and energetics.

The traffic infrastructure includes: road, rail, water and air traffic.

The water-economy infrastructure includes: systems for monitoring and controlling drinking water quality, systems for reducing waste water production, sewer channel network as well as subterranean waters.

The energetics infrastructure encompasses: the electro energetic transport and distribution network, energy objects, renewable energy sources, and the gas and distributive networks.

Social services include: education, health and social security.

The identified social aspects of development are measured and interpreted using the following basic indicators: poverty, spatial and social isolation, access to different institutions, demographic characteristics, infrastructure potentials as well as ecology.

Document [8] defines strategic factors which influence the development of the relevant region in APV, which are: basic geographic and historical data, environment, population, human resources, economy, agriculture, infrastructure, tourism and culture, education, health and social security.

The key development problems which characterize rural regions in APV, besides structural imbalance, are according to [8]: large number of unemployed citizens, non-utilization of existing production capacities, extensive agricultural production, social integration quality, ecological problems (air and water pollution, as well as wild landfills), adverse demographic trends of rural regions, insufficient tourism potential utilization, weak connection between education and development- reach institutions with economy, weak organization and insufficient education of EU application funds staff.

The development goals and strategies analyzed in [8], include three interconnecting priorities:

Smart growth: knowledge and innovation-based economic development;

Sustainable growth: the promotion of an economy which uses resources more efficiently, and is also more competitive and green-oriented.

Inclusive growth: the promotion of an economy which is characterized by a high employment rate and which achieves social and territorial cohesion.

In order to make the above-mentioned priorities applicable in practice, five key goals are identified: employment, education, culture and innovation, social inclusion and poverty minimization, battle against climate change and energetics.

The role of the SCORE ontology is to semantically present the identified socio-economic characteristics and development goals of APV. The social and economic status profiles which are analyzed in the CODEX project have been used as a basis for semantic presentation of development goals and priorities of the APV region.

\subsection{SCORE Ontology Conceptualization}

In accordance with the identified strategic characteristics of the analyzed domain (in previous section), the SCORE ontology will semantically present the socio-economic characteristics of the APV region. In the process of conceptualization based on the analysis from section 3.1 of this paper, three main ontological concepts have been identified: Spatial aspect, Development aspect and Strategic priority aspect. Figure 1 shows the conceptual model of the SCORE ontology.

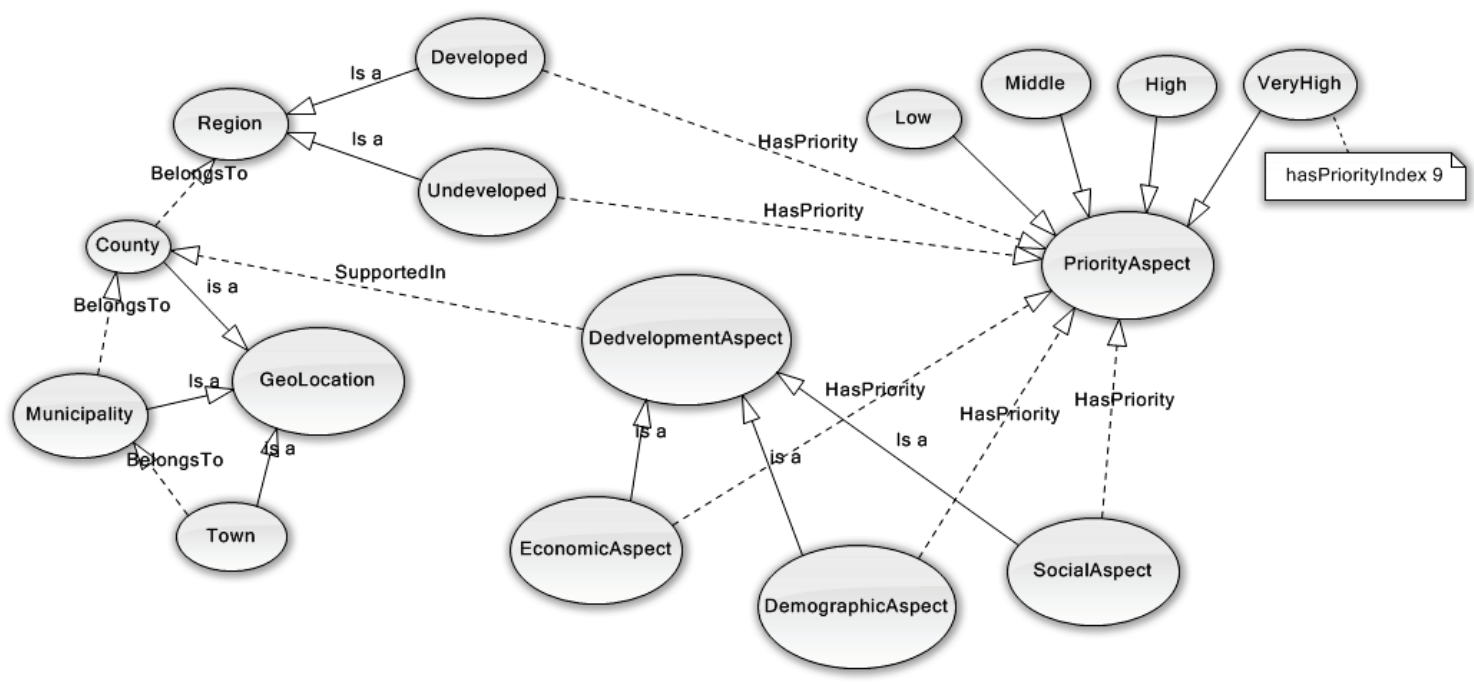

Figure 1 The SCORE ontology- conceptual model

The subconcepts identified within the main concepts will be described below.

\subsubsection{GeoLocation Concept}

The spatial aspect is described by the Region concept and includes populated places and their administrative organization (district, municipality) in APV.

\subsubsection{Region Concept}

This concept describes the economic division of regions and municipalities in APV into developed and undeveloped ones. This categorization is defined by state development strategies. 


\subsubsection{Development Aspect Concept}

The analyzed strategic documents are presented by the Development Aspect concept of the SCORE ontology. Three main development aspects are identified: economic, demographic and social.

\section{1) Ontological subconcept Economic}

This concept describes the economic development aspect and identifies industry areas: traffic, agriculture, tourism, crafts, trade and mining. Because the leading area in AP Vojvodina is agriculture, this area is analyzed in detail within the ontology. Different subclasses are identified within the Agriculture class: forestry, fishing, livestock and farming. The farming subclass defines subclasses which describe the production of industrial plants, grains, fruits and vine.

\section{2) Ontological subconcept Demographic}

This concept describes socio economic characteristics of a population which are expressed statistically, such as age, gender, education level, income level, marital status, occupation, religion, birthrate, death rate, average size of a family, average age at marriage.

\section{3) Ontological subconcept Social}

Within the social development aspects of state strategic documents, undereducated persons, rural population, refugees as well as disabled persons are separately identified.

\subsubsection{Priority Concept}

This concept defines priority levels of identified development aspects and grades every priority level. The ontology is, in this way, enabled to grade priorities of development aspects. Index values of the priorities should be given from professionals of the field in the process of ontology implementation.

This ontological prototype predicts four priority levels (Low, Middle, High and Very High) and every level has been assigned a value. The priority assessment and priority index value procedure will be described in detail in the following section.

\section{SCORE ONTOLOGY IMPLEMENTATION}

After defining taxonomy of basic concepts using the Protégé editor, ontology classes, class attributes, class instance attributes as well as class instances have been modeled, all of which have been identified in the conceptualization process and documented in the ontology.

There are two goals in the process of SCORE ontology implementation. The first is populating the ontology with instances belonging to regional and development concepts shown in Fig. 1. The second one is research-based numerical evaluation of the priority index value which is represented by the Priority concept within the ontology (Fig. 1).

The implementation process described in this paper has four stages:

Stage one is a socio-economic analysis which represents an analytical basis for starting a process of socio-economic development of the relevant region. This enables insight into the existing state, identification of the problem and developmental limits.

Stage two is characterized by a SWOT analysis of priorities [14]. The SWOT analysis utilizes data which have been identified during stage one of the implementation. Based on this data, matrices are created with entries such as strength, weakness, threats and chances for all key sectors, current socio-economic state of the region and all priorities of the regional infrastructure.

Stage three includes grading of built SWOTs for every analyzed region. The goal of such grading is to isolate priority weaknesses and relevant strengths of every defined development aspect ontologically described in the Development Aspect class. In this way, a basis for clearly defining priorities and development goals is created. This stage is a basis for assigning a numerical value to priority indices of every analyzed development aspect.

Stage four is characterized by the SCORE ontology population with instances, i.e. development priorities which are identified in the third stage of the ontology implementation. The next step is assigning properties to every instance. The properties represent development priorities defined in the third stage: HasPriority and BelongsTo.

When building the prototype model described in this paper, the SCORE ontology is populated with a few instances to which different properties have been assigned in order to represent the reasoning of the created ontology.

The Protégé editor has been chosen to generate the SCORE ontology, and the JAVA platform with JENA API for building the application and execution of SPARQL queries.

\section{A PROTOTYPE EXAMPLE OF SCORE ONTOLOGY USE}

This section describes a prototype example of SCORE ontology use. The prototype example will be presented with the help of a client case for which the support for the funding-approval process is presented. Because the goal of this example is to show the use of the SCORE ontology, priority index values are in this example given arbitrarily without prior realization of the implementation stages described in the lines above, and the ontology is populated with a few instances in order to show the reasoning of the created ontology. The client case is shown in Tab. 1.

Table 1 The client case

\begin{tabular}{|c|c|c|c|c|c|}
\hline Town & Age & Gender & $\begin{array}{c}\text { Purpose } \\
\text { of loan }\end{array}$ & $\begin{array}{c}\text { Industry } \\
\text { type }\end{array}$ & $\begin{array}{c}\text { Economic } \\
\text { status }\end{array}$ \\
\hline Uljma & 28 & Female & Machines & Handcrafts & Unemployed \\
\hline
\end{tabular}

After gathering the basic client data, the reasoning of the SCORE ontology and the process of decision proposal will be shown. Fig. 2 shows instances populated in the stage of ontology implementation and relations between proposed instances.

The result of A-box and T-box query execution over the ontology are priority index values whose sum is the decision proposal for funds placement. For the described 
client case, SPARQL execution gives the following conclusions:

The first group of queries refers to the identification of the geographic position of the client: the client lives in
Uljma, which is located in the province Vršac, the province Vršac belongs to the South Banat district, the South Banat district is an undeveloped region and has a priority of value 9 .

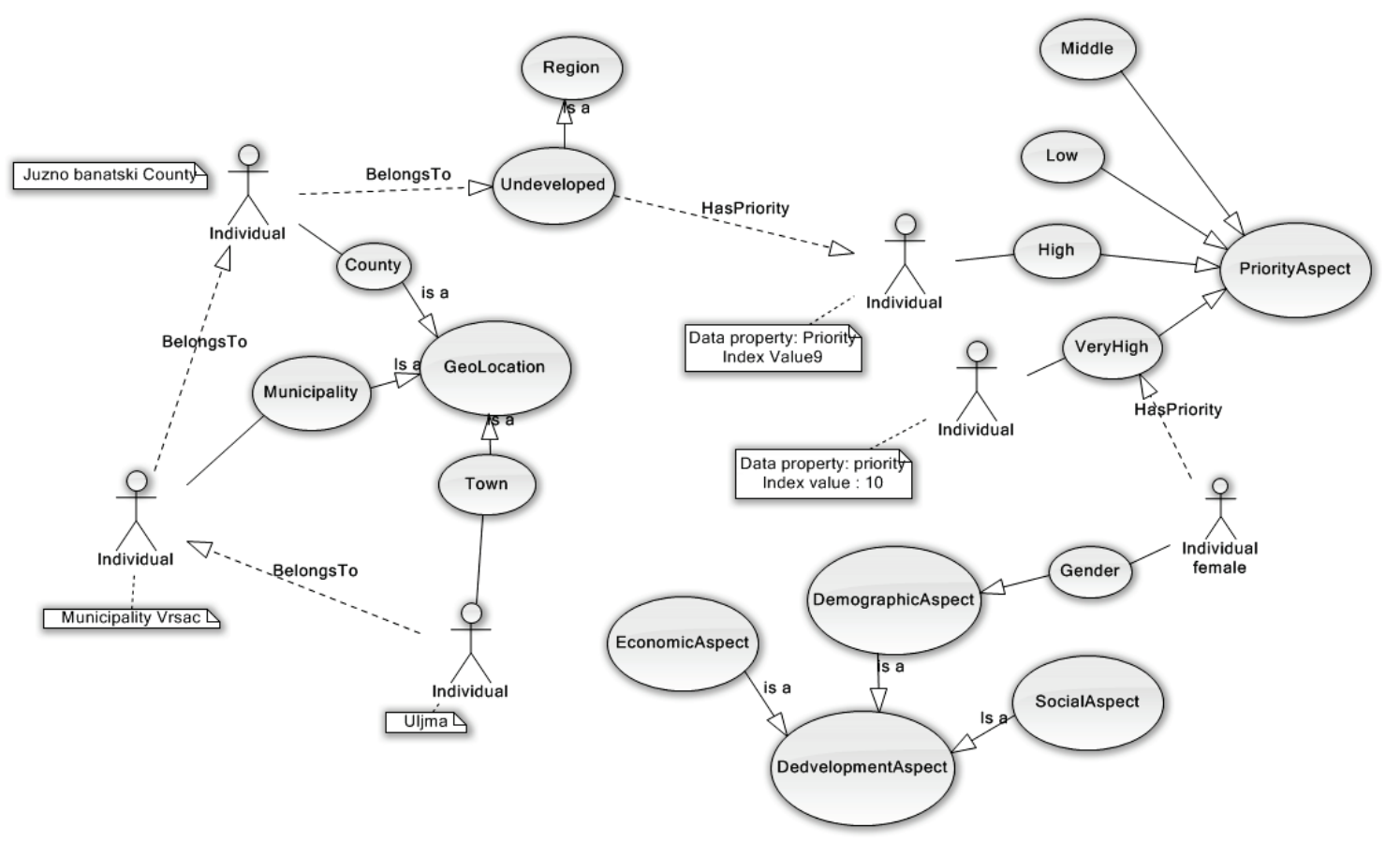

Figure 2 Excerpt of the SCORE ontology instance

The second group of queries analyses the client age: the client is classified into the group of young clients (demographic development aspect), young clients have Low priority of value 7 .

The third group of queries refers to the gender of the client (the demographic concept of gender structure). The client is female, clients which are female have a VeryHigh priority of value 10 .

The fourth group of queries refers to the economic aspect, for which the support of state development funds is requested: the client works in Handicrafts. This area is supported in the Vršac province. Handicrafts have Middle priority with priority index value 8 .

The fifth group of queries refers to the client employment status (SocialAspect). The client is unemployed. Unemployed clients have investment priority of value 9 .

Priority index values obtained via client case analysis defined in Tab. 1 are shown in Tab. 2.

Based on the cumulative score, a decision proposal on the placement of funds for a specific client case is generated. By using the SCORE ontology, a decision proposal will rule out non-objective decision making of administrative workers and expert-body members.

Table 2 The client score

\begin{tabular}{|c|l|c|}
\hline \multicolumn{2}{|l|}{ Parameters } & Priority index \\
\hline Town & Uljma & 9 \\
\hline Age & $18-30$ & 7 \\
\hline Gender & Female & 10 \\
\hline Industry type & Handcrafts & 8 \\
\hline Economic status & Unemployed & 9 \\
\hline & & $\mathbf{4 3}$ \\
\hline
\end{tabular}

The decision proposal will be created based on development strategies and realized socio-economic analysis implemented in the SCORE ontology.

By using the semantically described development strategies implemented in the SCORE ontology all government subsidies will be steered in the direction of strategic development of the analyzed region.

\section{CONCLUSIONS}

This paper separately analyzes specifics of decisionmaking support in the process of fund placement approval in state development funds. Research in this field has led to the creation of the SCORE ontology which represents a knowledge basis for DSS.

The proposed ontology encompasses conceptualized knowledge on strategic socio-economic characteristics which are valuable to the domain analyzed in this paper, as well as conceptualized knowledge which is a result of the socio-economic analysis of the relevant domain. In this way, the created ontology enables to grade identified development aspects of the analyzed domain and their implementation in the decision-making process.

The main advantage of using SCORE ontology is to describe the client in a concise description of the client case. Using the proposed method in government development funds we ensure the following advantages: Minimization of the investment risk; Focused global investments in accordance with the development strategy and Objective analysis-based decision making.

Future research should focus on evaluating the proposed SCORE ontology as well as its further development and popularization of the increasing number of the development characteristics as is defined in the 
state development strategies. Second direction of the future research should investigate and semantically present the mutual influence of the key strategic socioeconomic characteristics that are discussed in this paper.

\section{ACKNOWLEDGEMENTS}

Research in this paper was partially supported by the Ministry of Science and Technological Development of the Republic of Serbia, through project no. 171039.

\section{REFERENCES}

[1] Abu-Hanna, A. (1994). Multiple Domain Models in Diagnostic Reasoning, PhD Thesis (University of Amsterdam, Amsterdam, The Netherlands.

[2] Laskey, K. B. (2006). Decision Making and Decision Support.

[3] Birmingham, W. \& Klinker, G. (1993). Knowledge acquisition tools with explicit problem-solving models. The Knowledge Engineering Review, 8(1), 5-25. https://doi.org/10.1017/S0269888900000047

[4] Meersman, R. et al. (2010) (Eds.): OTM 2010 Workshops, LNCS 6428, 585-594. Springer-Verlag Berlin Heidelberg.

[5] Rospocher, M. \& Serafini, L. (2012). Ontology-centric Decision Support. Proc. SeRSy, 61-72

[6] Salles, M. (2010). Supporting Public Decision Making-A Progressive Approach Aided by Ontology. International Journal of Decision Support System Technology, 2(1), 2135. https://doi.org/10.4018/jdsst.2010101602

[7] Bogdanov, N. Mala ruralna domaćinstva u Srbiji i ruralna nepoljoprivredna ekonomija, http://www.undp.org.rs

[8] (2013). CODEX, Coordinated Development and Knowledge Exchange, on Spatial Planning Methodology, Project: HUSRB/1203/213/151.

[9] (2005). Law and the Semantic Web, Springer, Heidelberg, DE, 142-157.

[10] (2009). Protege, Ontology Editor and Knowledge Acquisition System, http://protege.stanford.edu/

[11] McGuinness, D. L., \& van Harmelen, F. (2007). OWL Web Ontology Language Overview, http://www.w3.org/TR/owlfeatures/.

[12] Sirin, E. \& Parsia, B. (2007). SPARQL-DL: SPARQL Query for OWL-DL. $3^{\text {rd }}$ OWL Experiences and Directions Workshop (OWLED-2007).

[13] Sirin, E., Parsia, B., Grau, B. C., Kalyanpur, A., \& Katz, Y. (2007). Pellet: A Practical OWL-DL Reasoner. Journal of Web Semantics, 5(2), 51-53. https://doi.org/10.1016/j.websem.2007.03.004

[14] Piercy, N. \& Giles, W. (1989). Making SWOT Analysis Work. Marketing Intelligence \& Planning, 7(5/6), 5-7. https://doi.org/10.1108/EUM0000000001042

[15] Glennon, D., Kiefer, N. M., Larson, C. E., \& Hwan-sik, C. (2007). Development and Validation of Credit-Scoring Models, Working Papers, 07-12, Cornell University, Center for Analytic Economics.

[16] Arsovski, S., Markoski, B., Pecev, P., Petrovacki, N., \& Lacmanovic, D. (2014). Advantages of using an Ontological Model of the State Development Funds. IJCCC, 2014.

[17] Syohei, I., Gehrmann, A., Minegishi, J., \& Nagai, Y. (2008). Ontology-Driven Decision Support Systems for Management System Audit. Proceedings of the $52^{\text {nd }}$ Annual Meeting of the ISSS / Madison.

[18] Arsovski, S., Markoski, B., \& Pecev, P. (2014). An approach to Ontology-based modeling of State Development Funds. Technical Gazette, 21(6), 1289-1296.
[19] Sram, N. \& Takács, M. (2015). An Ontology Model-based Minnesota Code. Acta Polytechnica Hungarica, 12(4).

[20] Prineas, R. J., Crow, R. S., Zhang, Z.-M. (2010). The Minnesota Code Manual of Electrocardiographic Findings, Springer-Verlag Berlin Heidelberg. https://doi.org/10.1007/978-1-84882-778-3

Contact information:

\section{Saša ARSOVSKI, PhD}

Imagineering Institute and City,

University of London

IDM Lab SdnBhd (1113259-P), Johor, Malaysia

E-mail: sasa@imagineeringinstitute.org

\section{Branko MARKOSKI, PhD}

University of Novi Sad,

Technical Faculty "Mihajlo Pupin", Zrenjanin

Republic of Serbia

E-mail: markonins@yahoo.com

\section{Nikola PETROV}

University of Novi Sad,

Technical Faculty "Mihajlo Pupin", Zrenjanin,

Republic of Serbia

E-mail: nikola.petrov@tfzr.rs

\section{Sanja STANISAVLJEV}

University of Novi Sad,

Technical Faculty "Mihajlo Pupin", Zrenjanin

Republic of Serbia

E-mail: Sanja.stanisavljev@tfzr.rs

\section{Mila ZAKIN}

University of Novi Sad,

Technical Faculty "Mihajlo Pupin", Zrenjanin,

Republic of Serbia

E-mail: mila.zakin@tfzr.rs 